\title{
The Neglected Importance of Sleep on the Formation and Aggravation of Facial Wrinkles and Their Prevention
}

\author{
Borut Poljsak1, Aleksandar Godic ${ }^{2}$, Andrej Starc ${ }^{1}$, Raja Dahmane1 \\ ${ }^{1}$ Faculty of Health Studies, University of Ljubljana, Ljubljana, Slovenia \\ ${ }^{2}$ University Hospital Lewisham, London, UK \\ Email: raja.dahmane@guest.arnes.si
}

Received 6 March 2016; accepted 29 May 2016; published 1 June 2016

Copyright (C) 2016 by authors and Scientific Research Publishing Inc.

This work is licensed under the Creative Commons Attribution International License (CC BY). http://creativecommons.org/licenses/by/4.0/

(c) (i) Open Access

\begin{abstract}
The duration of sleep and the position of the face while resting on a pillow have a negative impact on the facial skin appearance and may lead to the formation of sleep wrinkles. Sleep lines occur when there is repetitive, long-term tension on the facial skin, which pushes or pulls the skin in a direction that is perpendicular to the direction of the muscles of the face. These lines tend to be more vertically oriented than expression lines and can be found on the forehead, around the eyebrows, the eyes, the cheeks, the chin, and the nasolabial folds. Our studies revealed that the average reduction of wrinkles in total investigated area of the face (expressed as the density of wrinkle per surface skin) was approximately $12 \%$ after 28 days of sleep on a specially-designed pillow. The specially designed anti-wrinkle pillows eliminate the pressure on the cheeks, the eyes and the mouth during sleep. Many such pillows have been designed to reduce the aging process and to encourage users to sleep in specific positions. Evidence supporting the claim that a special pillow prevents wrinkles was presented. Nevertheless, prolonged human studies are required to further elucidate the role of sleeping on appearance of facial wrinkles.
\end{abstract}

\section{Keywords}

Sleep Wrinkles, Anti-Wrinkle Pillow, Facial Wrinkles

\section{Introduction}

The duration of sleep and the position of the face while resting on a pillow have a negative impact on the facial skin appearance and may lead to the formation of sleep wrinkles, which can develop into permanent sleep lines 
with time. Medical procedures, which cause facial muscle paralysis (botulinum toxin injections), and hyaluronic acid injections (dermal fillers) cannot alleviate sleep wrinkles, because they are not a consequence of the facial muscle contraction but the skin compression during sleep. Usage of an anti-wrinkle pillow seems to be a good strategy to prevent and improve sleep wrinkles.

Dr. Stegman published "Sleep Creases" in 1987 and was the first who demonstrated the aetiology of the sleep creases [1]. He described diagonal lines on the cheeks and the lower face and vertical lines on the forehead. Later it was noticed that sleep also aggravates nasolabial folds and "crow's feet" around the eyes. Dr. Stegman observed that sleep wrinkles are different than wrinkles caused by sun damage, smoking, frowning or laughing, since they are caused by specific sleep behaviour and if not resolved, deepen over time. His theory was later confirmed by Fulton and Gaminchi [2] and Sarifakioğlu et al. [3].

Many people sleep sideways; the skin in this position is squeezed and crushed against a pillow, which leads to the formation of compression wrinkles that become permanent as we age. The same happens when we sleep in the prone position facing a pillow. Sleep lines occur when there is repetitive, long-term tension on the facial skin, which pushes or pulls the skin in a direction that is perpendicular to the direction of the muscles of the face. These lines tend to be more vertically oriented than expression lines and can be found on the forehead, around the eyebrows, the eyes, the cheeks, the chin, and the nasolabial folds (Figures 1-3). Due to the weight of the head, the gravitational force becomes very aggressive and causes the muscles to elongate. Considering that most people sleep about one third of their life, prolonged and repeated distortion of the facial skin will eventually result in wrinkle formation. Nasolabial folds and wrinkles around the eyes are particularly noticeable in the morning after waking up from sleep. The depth of wrinkles usually reduces during the day because in the upright position gravity causes the cheeks to pull down on the skin around the eyes thereby reducing the wrinkles, which is even more noticeable at a younger age. However, continuous pressure on the face for a period of several hours may cause the formation of permanent wrinkles, which is even accelerated in elderly who have decreased skin elasticity and atrophy the facial bones and cartilage.

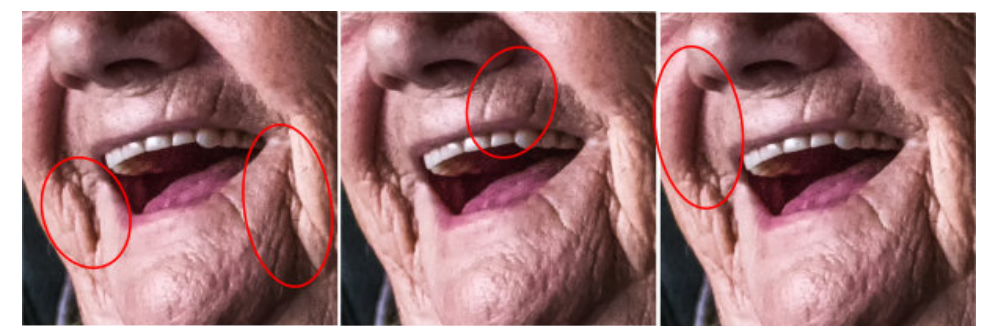

Figure 1. Deep vertical sleep creases around the lips, and nasolabial folds.

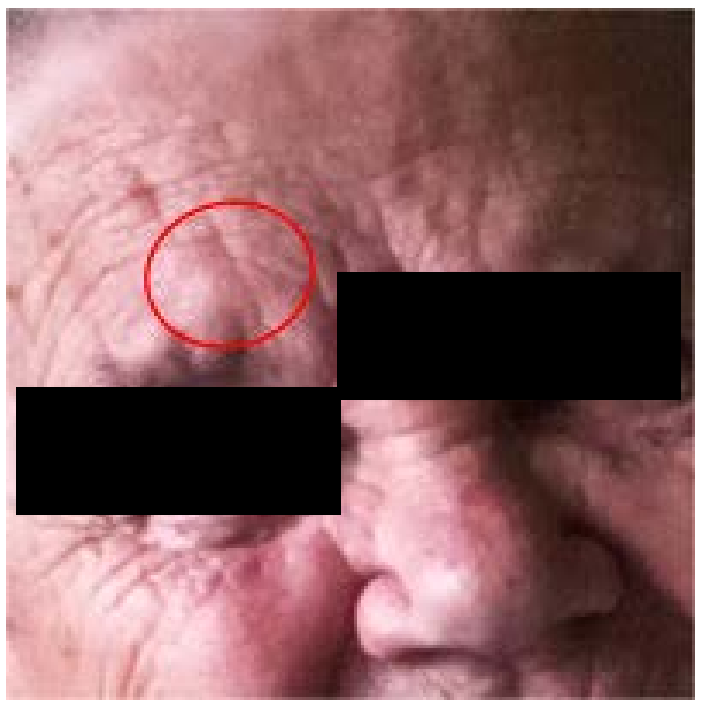

Figure 2. Deep vertical sleep creases around the forehead. 


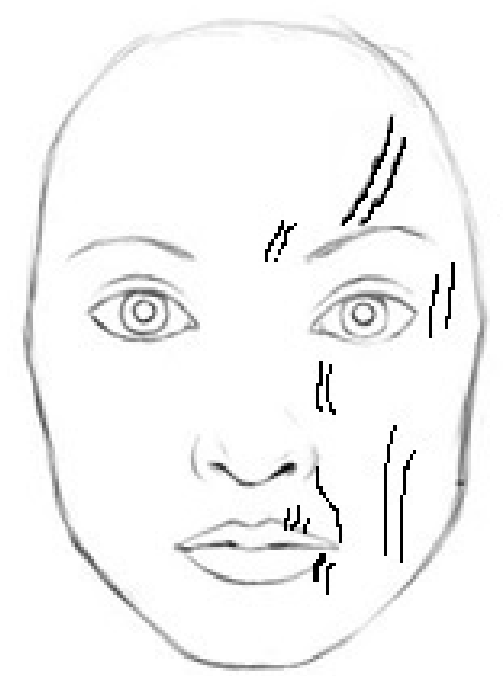

Figure 3. Schematic presentation of sleep lines, which are vertically oriented to the pulling and pushing forces on the face during sleep.

\section{Interventions to Alleviate Sleep Wrinkles and an Anti-Wrinkle Pillow}

Fulton and Gaminchi [2] reported that sleep lines are related to the facial attachments of the superficial musculoaponeurotic system and recommended release of these attachments with a carbon dioxide laser to achieve a good clinical outcome, which is not widely used in practice. Medical procedures, which cause facial muscle paralysis (botulinum toxin injections), and intradermal injections with hyaluronic acid cannot alleviate sleep wrinkles, because they are not caused by the facial muscle contractions but the skin deformations during sleep. Although fillers may not provide a long term solution Fulton et al. [2] have shown that subcision w/fat transfer does improve the appearance of these lines.

There are not many human trials related to sleep lines research. There is a lack of well-controlled study, with significant patient sampling and consistent results. Two smaller studies revealed that the severity of sleep lines could be reduced with an appropriate pillow or pillowcase. The study of Poljsak et al., [4] revealed that the average reduction of wrinkles in total investigated area of the face (expressed as the density of wrinkle per surface skin) was approximately $12 \%$ after 28 days of sleep on a specially-designed pillow. This study, however, has three important limitations: a small sample size, short duration (only one month), and lacks a control group. Contrary to this, Cotlus [5] did not find a significant correlation between sleep position and appearance of the facial wrinkles. Forty one "right-sided" and 23 "left-sided" participants were included in the study. Though this study also has several limitations, firstly, the author subjectively determined the sleeping position by asking participants whether they sleep mostly on the right or left side of the body. Secondly, the author neglected the fact that adults reportedly change their sleep position between three and 45 times per night with 10 to 12 different sleep positions used during the night. He did not consider other confounding factors, which contribute to wrinkle formation, like increased sun exposure of the left side of the face (and therefore reduced skin elasticity). Last but not least, the accuracy of his results can be questioned given the subjective assessments of wrinkles by only one independent physician. The study of Baek et al., [6] revealed that sleeping on copper oxide-containing pillowcases results in reduction of wrinkles depth and overall improvement of skin appearance. This effect, however, can be attributed more to copper-impregnated fibers and copper up-regulation and secretion of extracellular skin proteins that to the pillow design.

The specially designed anti-wrinkle pillows eliminate the pressure on the cheeks, the eyes and the mouth during sleep. Many such pillows have been designed to reduce the aging process and to encourage users to sleep in specific positions (Table 1). Usually they have a hollow depth to minimize and redistribute pressure on most exposed areas of the face to the chin and the forehead, thus reducing the formation of sleep wrinkles. They also elevate the face and do not press against the eyes and the mouth, thus reducing the formation of facial lines and wrinkles (Figure 4(a)), as reported above. Additionally, V-designed pillows minimize the face-to-pillow contact by aligning the neck and the spine (Figure 4(b)). 


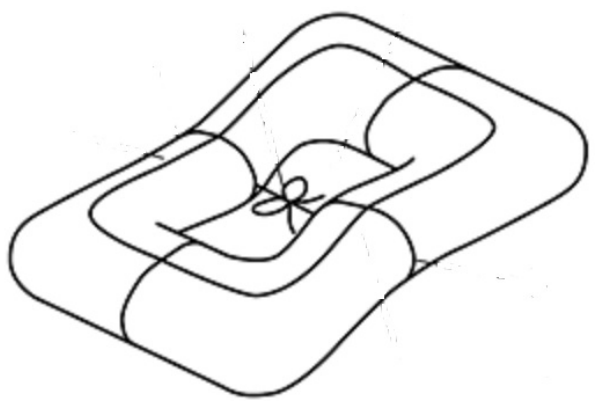

(a)

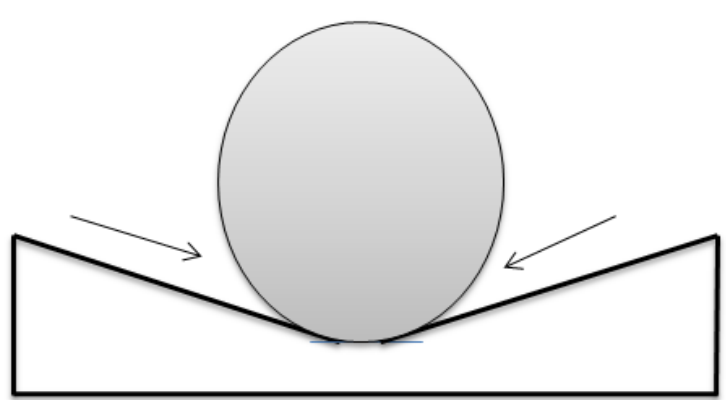

(b)

Figure 4. Example of the specially designed anti-wrinkle pillows that minimize the shear and compression forces on the face by: (a) hollow depth and (b) by V-design (side view).

Table 1. Different sleep pillows, which are reportedly efficient in minimizing the formation of sleep wrinkles.

\begin{tabular}{cc}
\hline Patent number & Pillow name \\
\hline US5054143 & Anti-wrinkle pillow \\
USD404237 & Anti-wrinkle pillow \\
USD410810 & Anti-aging pillow \\
USD434936 & Face pillow \\
USD474364 & Anti-wrinkle pillow \\
US7434281 & Pillow having anti-wrinkling properties \\
US8161588 & Anti-aging pillow \\
US 20030188383 & Pillow design to aid proper sleeping position which avoids facial wrinkle development \\
US2295906 & Pillow \\
US 5054143 A & Anti-wrinkle pillow \\
\hline
\end{tabular}

\section{Conclusion}

Evidence supporting the claim that a special pillow prevents wrinkles was presented. Nevertheless, prolonged human studies are required to further elucidate the role of sleeping on appearance of facial wrinkles.

\section{References}

[1] Stegman, S.J. (1987) Sleep Creases. American Journal of Cosmetic Surgery, 4, 277-280.

[2] Fulton Jr., J.E. and Gaminchi, F. (1999) Sleep Lines. Dermatologic Surgery, 25, 59-62. http://dx.doi.org/10.1046/j.1524-4725.1999.08073.x

[3] Sarifakioğlu, N., Terzioğlu, A., Ates, L. and Aslan, G. (2004) A New Phenomenon: "Sleep Lines" on the Face. Scandinavian Journal of Plastic and Reconstructive Surgery and Hand Surgery, 38, 244-247. http://dx.doi.org/10.1080/02844310410027257

[4] Poljsak, B., Godic, A., Fink, R., Oder, M., Lampe, T. and Dahmane, R. (2015) Sleeping on an Anti-Wrinkle Pillow Reduces Facial Wrinkles: Results from an Anatomical Study. Forensic Medicine and Anatomy Research, 3, 48-56. http://dx.doi.org/10.4236/fmar.2015.32010

[5] Kotlus, B.S. (2013) Effect of Sleep Position on Perceived Facial Aging. Dermatologic Surgery, 39, 1360-1362. http://dx.doi.org/10.1111/dsu.12266

[6] Baek, J.H., Yoo, M.A., Koh, J.S. and Borkow, G. (2012) Reduction of Facial Wrinkles Depth by Sleeping on Copper Oxide-Containing Pillowcases: A Double Blind, Placebo Controlled, Parallel, Randomized Clinical Study. Journal of Cosmetic Dermatology, 11, 193-200. http://dx.doi.org/10.1111/j.1473-2165.2012.00624.x 